\title{
Internacionalización de la Educación Superior y movilidad académica en la universidad pública uruguaya
}

\section{Internacionalização do Ensino Superior e mobilidade acadêmica na universidade pública do Uruguai} Internationalization of Higher Education and academic
mobility at the public university of Uruguay

Patricia Viera-Duarte ${ }^{1}$

Adriana Chiancone ${ }^{2}$

Enrique Martínez Larrechea ${ }^{2}$

DOI: http://dx.doi.org/10.20435/serie-estudos.v0i0.1397

\begin{abstract}
Resumen: En el artículo se propone describir las bases conceptuales con las que la Universidad de la República del Uruguay (Udelar) aborda el fenómeno de la internacionalización de la Educación Superior. Se intenta describir las políticas y los programas de inserción regional y de internacionalización en el período que va desde 1985 a 2019. El trabajo se organizó a partir de datos recogidos en el marco del Grupo Internacional de Estudos e Pesquisas em Educação Superior (GIEPES). Complementariamente, se empleó el análisis documental. Se considera que Universidad de la República desarrolló desde 1990 respuestas proactivas a los cambios del contexto regional y global que se expresaron en políticas y prácticas de internacionalización, las cuales priorizaron el contexto regional. Dada la peculiar conformación de la Educación Superior uruguaya, en la que la Udelar fue históricamente la única universidad pública, dichas políticas y prácticas contribuyeron a configurar la política nacional en la materia, así como configurar en forma estable algunos de los actuales mecanismos regionales en la Educación Superior. También se da cuenta de iniciativas de internacionalización e integración en la frontera uruguayo-brasileña. Se concluye en que la internacionalización de la Udelar es el resultado de su agenda política de transformación interna, desarrollada desde 1985, en la que se incluye el proceso de descentralización, especialmente relevante en la zona de frontera. Al mismo tiempo que permitía reconvertirse a la institución, fue también una respuesta a algunos desafíos externos estratégicos.
\end{abstract}

Palabras clave: Educación Superior; internacionalización; movilidad académica; integración regional; descentralización.

\footnotetext{
${ }^{1}$ Universidad de la República (UdelaR), Montevideo, Uruguay.

${ }^{2}$ Instituto Universitario Sudamericano (IUSUR), Montevideo, Uruguay.
} 
Resumo: O artigo propõe descrever as bases conceituais com as quais a Universidade da República do Uruguai (Udelar) aborda o fenômeno da internacionalização do Ensino Superior. É feita uma tentativa de descrever as políticas e os programas de inserção regional e de internacionalização no período de 1985 a 2019. O trabalho foi organizado com base em dados coletados no âmbito do Grupo Internacional de Estudos e Pesquisas em Educação Superior (GIEPES). Além disso, foi utilizada a análise documental. Considera-se que a Universidade da República desenvolveu desde 1990 respostas proativas às mudanças no contexto regional e global que foram expressas nas políticas e práticas de internacionalização, as quais priorizaram o contexto regional. Dada a conformação peculiar do Ensino Superior uruguaio, em que a Udelar foi historicamente a única universidade pública, essas políticas e práticas contribuíram para configurar a política nacional sobre o assunto, além de configurar de forma estável alguns dos atuais mecanismos de regionalismo no Ensino Superior. Também realiza iniciativas de internacionalização e integração na fronteira entre o Uruguai e o Brasil. Conclui-se que a internacionalização da Udelar é o resultado de sua agenda política de transformação interna, desenvolvida desde 1985, que inclui o processo de descentralização, especialmente relevante na área de fronteira. Ao permitir a conversão da instituição, foi também uma resposta a alguns desafios estratégicos externos.

Palavras-chave: Ensino Superior; internacionalização; mobilidade acadêmica; integração regional; descentralização.

Abstract: The article proposes to describe the conceptual bases with which the University of the Republic of Uruguay (Udelar) addresses the phenomenon of the internationalization of Higher Education. An attempt is made to describe the policies and programs of regional insertion and internationalization in the period from 1985 to 2019. The work was organized based on data collected within the framework of the International Group of Studies and Research on Higher Education (GIEPES) in addition to documentary analysis. It is considered that the University of the Republic developed since 1990 proactive responses to changes in the regional and global context that were expressed in internationalization policies and practices, which prioritized the regional context. Given the peculiar conformation of Uruguayan Higher Education, in which the Udelar was historically the only public university, these policies and practices contributed to configure the national policy on the subject, as well as stably configure some of the current regional mechanisms in Higher Education. It also realizes initiatives of internationalization and integration in the Uruguayan-Brazilian border. It is concluded that the internationalization of the Udelar is the result of its internal transformation political agenda, developed since 1985, which includes the decentralization process, especially relevant in the border area. While allowing the institution to be converted, it was also a response to some strategic external challenges.

Keywords: Higher Education; internationalization; academic mobility; regional integration; decentralization.

\section{INTRODUCCIÓN}

La Universidad de la República (Udelar) es la mayor institución de educación superior uruguaya y, durante mucho tiempo (entre 1833 y 1985), la única institución universitaria del país. Hoy es la primera de un limitado conjunto de siete universidades, entre las cuales dos son universidades públicas. 
La Universidad de la República precedió a la existencia de un sistema de educación superior, cumpliendo hasta el presente funciones sistémicas. Concentra gran parte de la investigación y de la docencia y un porcentaje relevante del gasto en ciencia, tecnología, innovación y educación superior. Por este motivo, la Udelar ha sido la matriz de la mayor parte de los núcleos de investigación científica, que desarrollan normalmente funciones de internacionalización basada en las disciplinas. Adicionalmente, ha sido la principal contraparte de la cooperación internacional en educación superior. A mediados de la década de los noventa y en un contexto de importantes cambios regionales e internacionales, la Udelar promovió una de las principales iniciativas subregionales de integración académica, a través de la Asociación de Universidades Grupo de Montevideo (en delante AUGM).

Desde la recuperación de su autonomía en 1985, la Udelar se planteó un proceso de transformación interna, que le permitiera hacer frente a su misión institucional. Entre esas transformaciones, muchas de ellas de gran alcance, se apuntó a dotar al rectorado de una estructura más ágil y de unidades especializadas de apoyo. El desarrollo de sus relaciones internacionales y de cooperación fue uno de los aspectos de ese ajuste de la estructura funcional.

A partir de 1991, el surgimiento del Sector Educativo del MERCOSUR implicó la activa participación de la Udelar en la delegación nacional, en relación con la temática de la educación superior y en la consiguiente elaboración de las políticas regionales del bloque en ese nivel. Además de diversos protocolos sobre educación superior, se avanzó el mecanismo experimental de acreditación (MEXA) y, más tarde, en el mecanismo definitivo que es el Sistema de Acreditación de Carreras Universitarias (ARCU SUR). Durante la década de los años noventa, promovió y construyó, junto a otras macro universidades públicas la AUGM.

La Udelar fue asimismo la principal contraparte de otras instancias de regionalización y de regionalismo en Educación Superior.

En algunos casos, estas acciones regionales implicaron la participación en programas de movilidad académica.

En las siguientes secciones, se aborda el concepto institucional de internacionalización; se describen las principales acciones, iniciativas y marcos institucionales que pautan la internacionalización de la universidad, revisando también los esquemas de movilidad académica principales. Ello permite 
dar un encuadre al análisis de los datos y describir mejor la gestión de la internacionalización y sus reformulaciones recientes.

Se analizan también las implicaciones del proceso de descentralización institucional en cuanto al surgimiento de otros formatos emergentes de internacionalización que pudieran identificarse en la actualidad. En este sentido se describen convergencias en materia de gestión de la internacionalización en la frontera terrestre con Brasil, entre las cuales se destacan algunas acciones hacia la búsqueda de convergencia en materia curricular y experiencias conjuntas de investigación y extensión. Finalmente, se presentan algunas conclusiones que pretenden conceptualizar la política de internacionalización desarrollada por la Universidad de la República desde la recuperación de su autonomía en 1985.

El trabajo se elaboró en base a los datos primarios, obtenidos a partir de la aplicación del cuestionario elaborado por el núcleo uruguayo del Grupo Internacional de Estudos e Pesquisas em Educação Superior (GIEPES). Se fundamenta también en el análisis de documentos institucionales.

La hipótesis de partida es que las políticas de internacionalización implementadas por la Universidad de la República se desarrollaron a lo largo de un proceso de más de tres décadas, mientras transcurría un proceso de transformación interna, en el marco de grandes transformaciones regionales y mundiales y como respuesta a ambas dimensiones, interna y externa. Dichas políticas resultaron centrales para el sistema de educación superior uruguayo y contribuyeron en un grado significativo a los esquemas regionales actualmente vigentes. En suma, la política de internacionalización de la Udelar es el resultado complejo de factores internos y externos. En primer lugar, de su agenda de transformación interna, desarrollada a partir de la recuperación de la autonomía en 1985, destacándose la relevancia del proceso de descentralización cumplido en la última década y que potenció su presencia en la frontera terrestre. Es también una respuesta a desafíos estratégicos del contexto externo.

\section{APUNTES SOBRE EL ABORDAJE INSTITUCIONAL EN RELACIÓN A LA INTERNACIONALIZACIÓN}

La internacionalización de la Educación Superior (ES), como respuesta al fenómeno de la globalización, se manifiesta de diferentes maneras en los diferentes contextos. América Latina ha apostado históricamente a una internacionalización 
de la educación superior con énfasis en la región, en el marco de un modelo de "cooperación solidaria", entendiendo a aquella como un bien público.

Altbach y Knight (2006) han contribuido a distinguir los conceptos de globalización y de internacionalización. Lo que suele denominarse usualmente como globalización es el proceso histórico, político, cultural y económico que se desarrolló en la década de los noventa, es decir a fines del siglo XX, acompañado por la difusión mundial de internet y por nuevos escenarios políticos y comerciales. La internacionalización comienza a resultar relevante, como respuesta proactiva, sea planificada o emergente, desde las instituciones de educación superior y de los países a los desafíos del contexto global.

Este contexto se caracterizaba por una nueva estructura hegemónica mundial, por los cambios en el estilo de desarrollo de América Latina hacia el regionalismo abierto, expresado en la firma del Tratado de Asunción que dio origen al MERCOSUR en 1991, y por los resultados de la Ronda Uruguay del Acuerdo General sobre Comercio y Aranceles (GATT), en inglés- que dio origen a la Organización Mundial de Comercio (OMC).

La institución no posee aun un documento de política, un strategy paper, adoptado oficialmente en la materia. Sin embargo, la entonces Ilamada Dirección General de Relaciones y Cooperación (DGRC) elaboró un informe de Internacionalización que contiene un abordaje del tema (DGRC, 2009). El informe reconoce como antecedentes del mismo, trabajos previos de la Dirección General de Relaciones y Cooperación (DGRC) , y dos eventos realizados en 2009, así como el Comunicado Final de la Conferencia Mundial de Educación Superior (UNESCO, 2009). El documento fue elaborado en el marco del proceso de debate para una "segunda" reforma de la Udelar, la cual no podría realizarse en forma descontextualizada de "Ios procesos internacionales en Educación Superior". La internacionalización: "Debe contribuir de manera crítica a sumarse a los sistemas mundiales y en la medida de las posibilidades sumarse e incidir en las transformaciones continentales y planetarias a la vez de ser crítica al interior de la institución" (DGRC, 2009, p. 1) .

\footnotetext{
${ }^{3}$ En adelante, las citas textuales se referirán a las diversas secciones del documento, indistintamente, con la mención de alguna de las siete páginas del documento, o en relación a las secciones temáticas del documento, que el lector puede ubicar en forma directa sin dificultad.
} 
Entre los procesos de carácter internacional que influyen en la educación superior, el documento destaca la movilidad universitaria, como uno de los fenómenos centrales. Sobre la universidad uruguaya se señala que "ha sido heredera de modelos universitarios europeos, aunque atenta y hasta promotora de diversas transformaciones de la Educación Superior. Muchas veces ha sido imitadora de otros modelos, pero también ha sido constructora más o menos crítica de un modelo propio y ha vivido envuelta en diversos escenarios internacionales" (DGRC, 2009, p. 1).

Entre estos escenarios se señala la importancia de la diáspora universitaria, conformada por científicos que "a su retorno post-dictadura tuvieron un papel trascendente en la reconstrucción universitaria".

La definición operativa sobre la internacionalización del documento señala que:

Vemos a la internacionalización como el necesario vínculo con la Educación Superior planetaria de forma de intercambiar experiencias desde lo académico a lo institucional construyendo o contribuyendo a construir una Educación Superior en el país y en el mundo (atendiendo naturalmente a las variaciones locales o regionales) sustentada en el desarrollo académico y científico, desarrollo que debe ser pertinente a las realidades locales en que las Universidades se insertan y que, a la vez, nutra y se vuelque sobre las sociedades en que se encuentran. (DGRC, 2009, p. 2).

Se asume a nivel conceptual de fondo la definición de internacionalización de Janet Knight (1994) que la caracteriza como "el proceso de integrar la dimensión internacional/intercultural en la enseñanza, la investigación y el servicio de la institución" (DGRC, 2009, p. 3).

La internacionalización, de acuerdo con el documento, favorece el desarrollo sociocultural y el entendimiento mutuo, y estimulando el surgimiento de alianzas estratégicas. El para qué de la internacionalización sería la mejora de la calidad de la educación y prepara a los actores universitarios para "desempeñarse en una comunidad multicultural y contribuye a la concreción de una sociedad del conocimiento".

De acuerdo con Miranda (2014) y con Morosini (2014), las actividades que constituirían la internacionalización son: la participación en redes y formas de organización de la cooperación y el intercambio; el reconocimiento de estudios y desarrollo curricular; la acreditación de calidad de programas e instituciones 
de educación superior; la movilidad académica en la cual se incluyen tanto la movilidad docente como estudiantil. Respecto de las redes, se señala como ejemplo paradigmático a la AUGM, reconociendo la relevancia de la Unión de Universidades de América Latina y el Caribe (UDUAL). Se considera importante en este aspecto también la participación en el Sector Educativo del MERCOSUR y en especial el Espacio Regional de Educación Superior (ERES).

El reconocimiento de estudios se considera un prerrequisito de la movilidad y como "instrumento para el aseguramiento de la calidad en el contexto de la internacionalización". En este sentido, se señala que: "el reconocimiento de períodos de estudios en el extranjero y de los créditos obtenidos, sigue sin tener una solución clara" al carecer de un reconocimiento automático de estudios. Una solución a esta situación podría ser la adopción del modelo desarrollado por la AUGM, en el marco del Programa ESCALA, en el que se acuerdan para cada caso de movilidad "un programa y sus respectivas acreditaciones", con base en la confianza entre instituciones (DGRC, 2009, p. 7).

Se indica asimismo que: "es importante considerar el desarrollo de programas académicos que aborden la atención de temas regionales o continentales, en particular favorecer el desarrollo de programas de estudios regionales, que otorgan títulos válidos en diversos países" (DGRC, 2009, p. 5). También se constata una carencia en materia de estudio de lenguas extranjeras.

Entre los temas aún pendientes de análisis y resolución, el informe de internacionalización señala: "el reconocimiento mutuo de títulos universitarios, la utilización de créditos académicos que sirvan como indicador del trabajo académico, la ejecución de programas de doble titulación y los de utilización conjunta", agregando que existen demandas de diversas disciplinas que impulsan programas cooperativos e internacionales (DGRC, 2009, p. 5).

Con referencia a la acreditación se subraya el rol del Sistema de Acreditación Regional de Carreras Universitarias de los Estados Partes del MERCOSUR y Estados Asociados (ARCUSUR) en el plano regional, en materia de movilidad, se subrayan los programas de movilidad en los que participa Udelar: PIMA-OEI; MARCAMERCOSUR; ESCALA-AUGM y Erasmus de la Unión Europea y los convenios, redes y otras formas de asociación, todos los cuales "aportan a nuestros estudiantes un enfoque intercultural y un aporte significativo en aspectos de formación académica, comprensión y respeto por otros pueblos y costumbres"... 
Los programas de movilidad enfrentan obstáculos para su exitosa implementación, entre los cuales el informe señala: "La falta de flexibilidad curricular y de mecanismos de transferencia de créditos, algunas trabas burocráticas (otorgamiento de becas, tasas aduaneras, etc.), el dominio insuficiente de lenguas extranjeras y el elevado número de estudiantes que trabajan. (DGRC, 2009, p. 6).

Los instrumentos disponibles en materia de internacionalización identificadas por el informe incluyen a los "instrumentos académicos y políticos basados en las estrategias de cooperación, la formación de redes, los foros y conferencias internacionales, el debate fecundo y de orientación solidaria" (DGRC, 2009, p. 2). En este sentido, se reconoce el aporte de la UNESCO, a través de diversas conferencias y declaraciones y los de diversas asociaciones de rectores y de universidades.

Dada su complejidad, surge como una de las primeras condiciones para la internacionalización, la gestión de ésta, a través de oficinas y unidades competentes de cooperación y relacionamiento. Se requiere el fortalecimiento de la Dirección General de Relaciones y Cooperación y la existencia de unidades de cooperación en los diversos servicios. En especial, se identifica como un aspecto clave el contar con una oficina "especializada en movilidad académica" y la inclusión de la temática de la cooperación internacional en la formación de recursos humanos en Udelar.

Se propone, finalmente, el reforzamiento del financiamiento de la internacionalización por parte del Estado, a fin de contar con mayores márgenes de libertad en la planificación de la cooperación, sus prioridades, temas y criterios.

\section{EL DESAFÍO DE LA GESTIÓN DE LA INTERNACIONALIZACIÓN}

Actualmente, el órgano responsable por la gestión de la internacionalización en Udelar es el Servicio de Relaciones Internacionales (SRI). El Servicio se encarga de gestionar los distintos programas de movilidad, la cooperación internacional multilateral y bilateral y analiza los convenios internacionales antes de la firma del Rector.

En el año 1988, se creó la Dirección General de Relaciones y Cooperación (DGCR) y su acción se orientó al fortalecimiento de la institución al medio, mientras que en el plano internacional se priorizó “la captación de docentes e investigadores 
provenientes del exterior, la formación de docentes e investigadores y la ejecución de proyectos conjuntos. [...] En este período hubo un aporte importante de organismos internacionales y agencias de países dispuestas a colaborar para la recuperación de los niveles de investigación y de recursos humanos calificados" (DGRC, 2019, p. 2).

En la siguiente década-1990- la universidad se insertó más activamente en iniciativas de internacionalización, entre las que se destacan el Programa ALFA de la Comisión Europea, el Programa de Cooperación Interuniversitaria de la Agencia Española de Cooperación Internacional (AECI) y la creación de la Asociación de Universidades Grupo Montevideo (AUGM).

Hasta mayo de 2018, la mencionada Dirección General de Relaciones y Cooperación de la Universidad de la República, actuó en estrecho contacto con una Comisión asesora del Consejo Directivo Central (CDC) y del Consejo Ejecutivo Delegado (CED) creada en el año 2007: la Comisión de Relaciones Internacionales y Cooperación Regional e Internacional (CRI-CRI).

La CRI y CRI estaba dotada de capacidades de iniciativa en materia de relaciones regionales e internacionales, políticas de cooperación internacional y regional, intercambio académico y convenios.

Conforme a la ordenanza de su creación, la Dirección General de Relaciones y Cooperación brindaba el apoyo administrativo y técnico necesario para el cumplimiento de sus fines.

Entre sus atribuciones se contaban el asesoramiento al CDC y al CED, la realización de propuestas sobre políticas institucionales que promuevan la inserción de la Universidad de la República en el ámbito internacional; la intervención en la definición de políticas presupuestales relacionadas con el relacionamiento externo y la cooperación universitaria y con la creación y adjudicación de fondos destinados a financiar actividades en estas materias.

También le competía actuar como organismo de orientación y coordinación de la Cooperación Internacional Universitaria y asesorar en materia de convenios internacionales. Le correspondía la propuesta de prioridades respecto a programas, proyectos, planes y actividades, el seguimiento, supervisión y evaluación de los proyectos y acciones en ese ámbito internacional (UDELAR, 2016 ${ }^{4}$ ).

\footnotetext{
${ }^{4}$ Disponible en: http://cooperacion.udelar.edu.uy/es/?page_id=178. Acceso el: 10 abr. 2018.
} 
En el año 2018, se crearon nuevas estructuras: una Presidencia del Servicio de Relaciones Internacionales (de carácter unipersonal), quien preside asimismo la Comisión cogobernada CRI y CRI; la Dirección de División Relaciones Internacionales y Cooperación Académica, con un Departamento de Convenios; la Dirección de Departamento de Programas y Proyectos; la Dirección de Departamento de Relaciones e Integración Regional con un personal compuesto por siete Técnicos, cinco Administrativos y un pasante, además de los directores correspondientes a cada sector.

El actual Servicio de Relaciones Internacionales (SRI) de la Udelar ha definido algunas áreas prioritarias, entre las que se destacan: la movilidad académica docente, movilidad estudiantil, cooperación académica internacional y acreditación regional de carreras prioritariamente en Ciencias básicas e Ingenierías.

\section{PROCESOS DE MOVILIDAD ACADÉMICA EN UDELAR}

La movilidad académica a nivel de docentes y técnicos se desarrolla en diversos marcos y programas de cooperación, como puede verse en la tabla a continuación, entre los que cabe mencionar ESCALA Docente-AUGM, MARCA (docentes), MARCA (coordinadores), ECOS (Francia), CAPES (Brasil) FUNDACIÓN CAROLINA (España), Programa 720 (contrapartida de convenios), Asociación Universitaria Iberoamericana de Posgrado (AUIP) ${ }^{5}$.

\subsection{Movilidad docente y del personal técnico}

De acuerdo con registros del 2016, la movilidad en un sentido u otro involucraba a unas doscientas personas.

\footnotetext{
${ }^{5}$ La información de la página web correspondiente en la Universidad de la República denomina "movilidad académica" la de los docentes y técnicos, distinguiéndola de la movilidad estudiantil. A nivel conceptual, en cambio, denomina movilidad académica tanto la docente como la estudiantil.
} 
Tabla 1- Movilidad de docentes y servidores técnicos

\begin{tabular}{|l|c|c|c|}
\hline \multicolumn{1}{|c|}{ Programa } & Recibidos & Enviados & $\begin{array}{c}\text { Total de } \\
\text { movilidades }\end{array}$ \\
\hline ESCALA Docente (AUGM) & 27 & 23 & 50 \\
\hline MARCA (docentes) & - & 5 & 5 \\
\hline MARCA (coordinadores) & - & 3 & 3 \\
\hline ECOS (Francia) & 13 & 16 & 29 \\
\hline CAPES (Brasil) & s/d & 4 & 4 \\
\hline FUNDACIÓN CAROLINA (España) & - & 3 & 3 \\
\hline $\begin{array}{l}\text { Programa 720 (contrapartida de convenios - } \\
\text { DGRC) }\end{array}$ & 52 & 50 & 102 \\
\hline AUIP & 4 & 7 & 11 \\
\hline TOTAL & $\mathbf{9 6}$ & $\mathbf{1 1 1}$ & $\mathbf{2 0 7}$ \\
\hline
\end{tabular}

Fuente: Sitio oficial Dirección General de Relaciones y Cooperación (UDELAR, 2016).

\subsection{Movilidad estudiantil}

La movilidad de estudiantes se desarrolla a través de diversos programas, tales como Cooperación y Convenios. Programas de Intercambio: Programa Erasmus Mundus action2; Erasmus+; ESCALA-AUGM- PAME; PIME; MARCA. Programa de Becas de Grado Iberoamericana.

Programa ESCALA de Estudiantes de Grado-AUGM. El programa fue creado en 1998, como una de las iniciativas estratégicas centrales de AUGM, una red que busca contribuir a la gestación de un espacio académico e intercultural común ampliado. Los estudiantes de grado regularmente matriculados en una universidad del Grupo pueden cursar parte de sus estudios durante un semestre lectivo en otra universidad de un país diferente al de su residencia, mediante el reconocimiento académico de los estudios cursados en la universidad de destino, como parte del cumplimiento del plan de estudios de su carrera. Entre sus objetivos se encuentran la "construcción de un espacio académico común regional a través de la movilidad de estudiantes, con la convicción de que, mediante la interacción y la convivencia entre alumnos y docentes de distintas universidades y países, no sólo se promoverá el intercambio académico y cultural sino también un mejor conocimiento de la diversidad y particularidades de los diferentes sistemas de educación superior instalados", así como la promoción 
de la integración regional y a la internacionalización de la educación superior regional "mediante la construcción de un espacio ampliado e internacional de movilidad e intercambio". También se apuesta al fortalecimiento institucional y al establecimiento de alianzas estratégicas y el desarrollo de actividades de intercambio entre las universidades de AUGM ${ }^{6}$.

El Programa Erasmus Mundus apunta a fomentar la cooperación entre instituciones de educación superior de la Unión Europea y terceros países, a través de la movilidad orientada a la realización de estudios de grado y de posgrado, así como del personal académico. UdelaR ha participado históricamente en varios consorcios de Erasmus: Mundus 17, Monesia, Erasmus Mundus 17, Babel y Preciosa. Cada proyecto está integrado por instituciones europeas y latinoamericanas ${ }^{7}$.

Erasmust es el nuevo programa para el período 2014-2020. Promueve diverso tipo de acciones claves (Key Actions): "KA1-Movilidades individuales (de corta duración, con créditos para estudiantes, docentes y personal académico) y Maestrías conjuntas. KA2-Cooperación para la innovación y buenas prácticas (Capacity Building actions). KA3-Apoyo a las políticas de reforma. Erasmus+ integra y reemplaza a los anteriores programas ERASMUS MUNDUS, ALFA, TEMPUS, EDULINK y otros de cooperación bilateral" ${ }^{8}$.

El Programa Académico de Movilidad Estudiantil de la Unión (PAME-UDUAL) está orientado a fortalecer el intercambio estudiantil de pregrado y fue aprobado por el Consejo Ejecutivo de la UDUAL en 2003. El programa "promueve la movilidad recíproca de estudiantes, entre instituciones de educación superior de la Unión, seleccionadas aleatoriamente. Para ello, las instituciones proponen el programa o programas educativos que consideran de alto nivel académico, en los que pueden recibir estudiantes para cursar actividades académicas regulares, mutuamente convenidas". Las instituciones deben ofrecer tres plazas académicas, una de cobertura amplia y dos de cobertura parcial, a estudiantes regulares de grado de alto desempeño que hayan promediado la formación de grado?

\footnotetext{
${ }^{6}$ Disponible en: http://cooperacion.udelar.edu.uy/es/?page_id=294

${ }^{7}$ Disponible en: http://cooperacion.udelar.edu.uy/es/?page_id=297

${ }^{8}$ Disponible en: http://cooperacion.udelar.edu.uy/es/?page_id=4692

${ }^{9}$ Disponible en: http://cooperacion.udelar.edu.uy/es/?page_id=301
} 
El Programa de Intercambio y Movilidad Académica (PIMA), de Organización de los Estados Iberoamericanos (OEI), es una iniciativa estratégica en el campo de la educación superior que intenta contribuir al desarrollo del Espacio Iberoamericano del Conocimiento (EIC). Los estudiantes realizarán en otro país iberoamericano un semestre académico de estudios, reconocido por su universidad de origen. Se busca "fortalecer la cooperación interuniversitaria y fomentar el desarrollo de la dimensión iberoamericana de la enseñanza superior mediante el desarrollo de proyectos multilaterales de intercambio de estudiantes de grado en la región"10.

En el marco iberoamericano, Udelar participa asimismo en dos redes académicas $^{11}$. El Programa de Movilidad Académica Regional se enfoca en los cursos acreditados por el mecanismo de acreditación de carreras de grado en el MERCOSUR (MARCA). Se trata del primer programa de movilidad de estudiantes de grado, a lo largo de un semestre académico promovido por el Sector Educativo del MERCOSUR, con los objetivos de contribuir a la mejora de la calidad de la enseñanza superior en las carreras acreditadas MERCOSUR y estimular la cooperación interinstitucional e internacional, así como: "Promover el conocimiento recíproco sobre la formación académica de las carreras de grado de los países del MERCOSUR y el perfeccionamiento de los procesos de formación, a través del intercambio de alumnos, docentes, investigadores y gestores/coordinadores de las carreras que participan del Mecanismo de acreditación de calidad MERCOSUR"12.

El programa busca también potenciar las experiencias de movilidad, conocer mejor los sistemas de evaluación en la región, el conocimiento de las lenguas oficiales del MERCOSUR y promover el reconocimiento de materias, grados, estudios y diplomas,

El Programa "Becas Iberoamérica. Estudiantes de Grado. Santander Universidades se desarrolló en el período 2011-2015, con el propósito de promover

${ }^{10}$ Disponible en: http://cooperacion.udelar.edu.uy/es/?page_id=299

${ }^{11}$ Red Desarrollo Agrario y Económico: Tendencias Actuales con la Universidad de Almería, España (Universidad coordinadora), Universidad del Mar, Chile, Universidad de Luján, Argentina y Universidad del Estado de Santa Catarina, Brasil.

Red Humboldt-Historia con la Universidad Pablo Olavide, España (Universidad coordinadora), Universidad del Norte, Colombia, Universidad de Guadalajara, México.

${ }^{12}$ Disponible en: http://cooperacion.udelar.edu.uy/es/?page_id=303 
la movilidad e intercambio de estudiantes entre universidades iberoamericanas, en el marco del Espacio iberoamericano del conocimiento. En el Segundo Encuentro Internacional de Rectores de Universia, celebrado en Guadalajara 2010 (México), el Banco Santander asumió el compromiso de iniciar el Programa, con "1.000 becas en su Convocatoria 2011, cuyo número será incrementado en 1.000 becas adicionales en sucesivas convocatorias anuales, alcanzando en 2015 un total de 5.000 becas" de 3.000 euros o el importe equivalente en dólares en la fecha de entrega ${ }^{13}$.

Según datos de 2016, este tipo de movilidad alcanzó a unas 150 personas, con algún predominio de las personas recibidas (82) sobre las enviadas (68). (Ver la tabla 2, a continuación).

Tabla 2- Movilidades de estudiantes registrada (2016)

\begin{tabular}{|l|c|c|c|}
\hline \multicolumn{1}{|c|}{ Programa } & Recibidos & Enviados & Total de movilidades \\
\hline $\begin{array}{l}\text { ESCALA } \\
\text { (AUGM) }\end{array}$ & 32 & 31 & 63 \\
\hline PIMA (J. de Andalucía) & - & 3 & 3 \\
\hline $\begin{array}{l}\text { BECAS IBEROAMÉRICA } \\
\text { Estudiantes de Grado }\end{array}$ & - & 2 & 29 \\
\hline $\begin{array}{l}\text { Escala de Estudiantes de } \\
\text { Posgrado (PEEP) (AUGM) }\end{array}$ & 15 & 14 & 16 \\
\hline MARCA. Estudiantes & 9 & 7 & 8 \\
\hline PAME & 4 & 4 & 28 \\
\hline ERASMUS MUNDUS & 22 & 6 & 1 \\
\hline $\begin{array}{l}\text { ERASMUS + } \\
\text { (Proyecto MOBILE+) }\end{array}$ & - & 1 & $\mathbf{1 5 0}$ \\
\hline TOTAL & $\mathbf{8 2}$ & $\mathbf{6 8}$ & $2016)$ \\
\hline
\end{tabular}

Fuente: Sitio oficial Dirección General de Relaciones y Cooperación (Udelar, 2016)

Como se puede ver, la principal política para promover el envío de estudiantes al exterior es el Programa ESCALA que posibilita cursar un semestre en universidades públicas de la región. Además, hay convenios de cooperación bilateral que incluye la movilidad estudiantil para la realización de un semestre en otras universidades que no pertenecen a AUGM, pero con las que se ha firmado

${ }^{13}$ Disponible en: http://cooperacion.udelar.edu.uy/es/?page_id=292 
convenios. Para los estudios de posgrados existe un Programa Escala, al que se suman otros instrumentos, como el "Programa Ecos", con unidades académicas de Universidades francesas.

Si bien en este momento no se cuenta con una cifra actual de número de estudiantes estudiando en otros países, sí se sabe que entre el 2005 y el 2016 había 559 estudiantes de Udelar estudiando en el exterior. De esos 559, unos 361 fueron estudiantes de grado y 198 de posgrado, dato que da cuenta de la mayor importancia que se le ha dado a la movilidad estudiantil de grado.

\section{FORMATOS DE INTERNACIONALIZACIÓN Y TRANSFORMACIÓN INTERNA}

La Universidad de la República, se ha embarcado en transformaciones que han recibido distintas denominaciones por parte de sus actores institucionales. A este proceso de cambio, le han llamado "la auto reforma" o segunda reforma haciendo alusión a la primera inspirada en Córdoba 1918.

Según Artigas (2015, p. 47),

[...] durante el transcurso del siglo XX la literatura histórica y los documentos más recientes registran un continuo de procesos institucionales en la República que recibió diversas denominaciones: "reforma" (a inicios del siglo), "cambio" (entre los años 50-60), transformación" (años 90 e inicios de 2000) y, a partir de 2006, nuevamente se plasma en el discurso universitario el término "reforma y, en este caso, "segunda reforma".

Uno de los ejes del cambio actual está abocado a la descentralización universitaria. Es así, que, en los últimos quince años y tras el objetivo de consolidar la equidad geográfica y social, se han puesto en marcha varias políticas de expansión en un planificado proceso de descentralización por regiones en el interior del país (UDELAR, 2012; UDELAR-CCI, 2015; DIARIO NORTE, 2018).

En la actualidad ya están conformados dos de los Centros Regionales Universitarios planificados para interior del país: el CENUR del Litoral (que además es una región localizada en frontera con Argentina) y el CENUR del Este. Todo este proceso ha sido muy cuidadoso de la calidad y bastante lento, particularmente en lo que se ha llamado la Región Noreste (territorio que hace frontera con Brasil), adonde hasta la fecha se opera desde sedes locales bien asentadas a las que les ha tomado más tiempo encontrar identidad regional y visión compartida como para constituirse en CENUR. 
Es de hacer notar que Uruguay es un país de pequeña dimensión territorial, por lo que casi todas sus regiones hacen frontera con países limítrofes. Con esta peculiaridad, resulta verosímil la hipótesis de que el proceso de expansión universitaria y de descentralización haya contribuido a caminar hacia experiencias de nuevas modalidades de internacionalización en sus sedes de frontera, principalmente en aquellas regiones donde existen sedes universitarias radicadas en "frontera seca" entre Uruguay y Brasil donde el flujo de intercambio -entre las sedes universitarias de uno y otro país- se daría de forma natural (IDR, 2018).

Para todo el interior del país, se comenzó a consolidar acciones tendientes hacia la descentralización a través de la creación de unidades académicas llamadas $\mathrm{PDU}^{14}$. En la región noreste en particular se viene desarrollando una oferta educativa con diversas carreras de tecnicaturas, licenciaturas e ingenierías, en algunos casos coorganizadas con otras instituciones de Educación Pública Superior u otro tipo de organizaciones que se radican en cada localidad. Es en estas sedes universitarias ubicadas en la frontera noreste ${ }^{15}$ donde comienzan a aparecer proyectos educativos que se han enriquecido de forma relevante a partir del intercambio con universidades del sur de Brasil. Esta coyuntura ha posibilitado avanzar tímidamente hacia otras modalidades de internacionalización en movilidad estudiantil-en estudios de grado y formación permanente- en movilidad docente a través de proyectos de posgrados y de investigación; y principalmente en movilidades conjuntas-de discentes, docentes y otros actores externos a Udelaren proyectos binacionales de extensión ${ }^{16}$ (VIERA DUARTE, 2018; 2017; OLVERA; VIERA, 2015; DINIS; MOURA DE MELLO, 2015).

Seguramente, han sido la iniciativas estudiantiles locales -a través del Centro de Estudiantes de las sedes de frontera de Udelar y de Unipampa- y los proyectos de Extensión, los pioneros en materia de "modalidades fronterizas

${ }^{14}$ En la Región Noreste de Udelar se han creado por concurso 16 Polos de desarrollo Universitario (PDU)

${ }^{15}$ El Centro Universitario de Rivera (CUR) y la Casa de la Universidad de Cerro Largo (CUCel) están ubicados en Departamentos limítrofes con Brasil.

${ }^{16}$ Actualmente se realiza el seguimiento de lo que dimos en llamar "nuevos formatos de internacionalización" que surgen en el proceso de implementación de las políticas de democratización universitaria y de descentralización de la Udelar. 
de internacionalización" y han logrado avances muy significativos en estas experiencias locales/internacionales ${ }^{17}$ aunque, debido el modo natural en el que se dan los hechos en una cultura periférica, existe poca documentación al respecto y no todas figuran como datos en los registros estadísticos oficiales.

La proyección a futuro hace pensar en el surgimiento de próximas transformaciones, ya que esta región de frontera noreste de Udelar, se encuentra transitando hacia la conformación de un nuevo CENUR y en este proceso todos los Polos de Desarrollo Universitario (PDU) se reconvertirán en otro tipo de unidad académica que necesariamente debe abandonar la lógica de "sedes locales con alta dependencia de las Facultades" para avanzar hacia una perspectiva regional en el interior. En esta coyuntura, se explica cierta tensión entre la posibilidad de que estas nuevas unidades ganen autonomía respecto a las Facultades (hoy Servicios de Referencia Académica radicados en la capital del país) o por el contrario que la lógica vertical de Facultades ceda espacios a la lógica regional de organización más horizontal. En este segundo escenario podría darse que el espacio de autonomía se logre por iniciativa y protagonismo activo del cogobierno de la región; si así ocurriera, entonces se potenciaría la impronta de frontera con nuevas formas de movilidad que son propicias para los intercambios académicos internacionales

${ }^{17}$ De acuerdo con el informe González (2018), las acciones tendientes a fortalecer los vínculos en materia de actividades de Extensión y Relaciones con el Medio en Frontera tuvo varios antecedentes. Entre el 2008 y 2018 se ejecuta un Programa de Desarrollo Sustentable de la Frontera Brasil-Uruguay, con el objetivo de generar las bases académicas de planificación territorial para el desarrollo sustentable en la región de frontera Brasil-Uruguay. En el proyecto participan los equipos de docentes e investigadores de distintas disciplinas, representantes de varias universidades públicas del estado brasileño de Río Grande do Sul La participación de la Udelar se ha dado bajo la coordinación de la Comisión Sectorial de Extensión y Actividades en el Medio (CSEAM), con apoyo de casas y centros universitarios de la región noreste A estos antecedentes le sigue el Proyecto de "Fortalecimiento interuniversitario en el área de Frontera con Brasil" (2016 - 2017) que se llevó a cabo con el objetivo de ofrecer formación y extensión para estudiantes de agronomía del Instituto EDUCAR, con participación de estudiantes y docentes de la Udelar del Instituto EDUCAR (MST) y Universidad Federal da Fronteira Sul (UFFS), Brasil. Se organizan encuentros Binacionales como el de Extensión Rural UFSM-Udelar coordinado por Facultad de Veterinaria (Udelar), con apoyo de CENUR Noroeste (sede Salto-Udelar), y del Centro Universitario de Rivera (Udelar). La iniciativa de internacionalizar la Extensión Rural es coordinada en conjunto con referentes de la Dirección General de Desarrollo Rural del Ministerio de Ganadería Agricultura y Pesca (MGAP) y con referentes de los técnicos que desarrollan libre ejercicio de la profesión. 
desde este locus estratégico para la producción y gestión del conocimiento con impacto en toda la Región desde una perspectiva geopolítica ${ }^{18}$.

De acuerdo con Bizzózero (2018) existen significados acerca de la internacionalización desde los que se puede visualizar una competencia geoeducativa y cultural en regiones periféricas. Si bien esta idea se había planteado desde otro nivel-pensada desde el papel de los socios pequeños en la conformación de los regionalismos a nivel de los Estados (BIZOZZERO; ABREU, 2000) ${ }^{19}$ - cabe un análisis similar, pero a nivel institucional: las regiones periféricas de las instituciones (como las sedes de frontera) pueden jugar un papel preponderante dentro de los procesos de conformación de una nueva estructura institucional en la creación de otras formas de movilidad académica. La hipótesis que aquí se maneja es que las políticas de descentralización pudieron causar modificaciones en los significados de la internacionalización universitaria. Basta pensar que, si no se hubiera desatado el proceso de descentralización territorial, no existirín hoy las sedes universitarias en las zonas de frontera en las que se identifican convergencias en materia de gestión, organización internacional y convergencias en materia curricular transfronteriza que se presentan como "otras formas de movilidad" (BIZOZZERO; ABREU, 2000; BIZOZZERO, 2018; BIZOZZERO; HERMO; MARTÍNEZ LARRECHEA, 2014).

Si bien hay que reconocer que existen antecedentes importantes a nivel de acuerdos bilaterales que han posibilitado el trabajo académico binacional, habría que reconocer el potencial local para generar nuevas modalidades de aprendizaje y circulación de saberes en estas sociedades que comienzan a ganar espacios de autonomía y de apertura internacional al mismo tiempo.

\footnotetext{
${ }^{18}$ Las ciudades de Sant'Ana do Livramento/Brasil y Rivera/Uruguay, forman una conurbación social, cultural y urbana de más de 160 mil habitantes que equidista en aproximadamente $500 \mathrm{Km}$ de Porto Alegre, Montevideo y Buenos Aires (ASCONAVIETA DA SILVA; SOUZA LIMA, 2015). Han sido nombradas por sus correspondientes parlamentos como "Ciudad Símbolo de la Integración del Mercosur".

${ }^{19}$ Para Bizzózero e Abreu (2000), Uruguay cumple un rol destacado en el MERCOSUR y no es de llamar la atención que siendo un país pequeño juegue un papel importante en lo que podría llamarse nuevas formas de regionalismo; de la misma forma que los países miembros de BENELUX tuvieron un rol destacado en el proceso de integración europea, condicionada por su evolución histórica, su ubicación geográfica y contexto geopolítico.
} 
Udelar es asiento de diversas cátedras UNESCO: Ias de Educación en Derechos Humanos, creada en 2002; de Manejo Costero Integrado del Cono Sur; de Agua y Cultura; de Carnaval y Patrimonio 20.

Entre los nuevos formatos o temas globales presentes en la internacionalización de la Udelar, cabe subrayar el reciente establecimiento de un Instituto Confucio en la Universidad de la República. Se trata de un centro de enseñanza del idioma Chino Mandarín, de difusión de la cultura china en Uruguay y de intercambio y de cooperación en educación y cultura entre Uruguay y China. Fue inaugurado en diciembre de 2017 y comenzó a impartir enseñanza en 2018. El convenio de base fue establecido entre la Universidad de la República y la Universidad de Qingdao y la Sede Central de los Institutos Confucio ${ }^{21}$.

\section{CONSIDERACIONES PROVISIONALES}

El proceso de transformación institucional iniciado en 1985 -con la recuperación de su autonomía y el regreso al país de numerosos científicos que contribuyeron a la visibilidad internacional de la ciencia local y a la internacionalización de los grupos de investigación en Udelar- es una dimensión clave para comprender las políticas de integración regional y de internacionalización de esta universidad. Adicionalmente, se enfrentó a un contexto externo que formulaba nuevos desafíos, entre ellos, determinar cuál sería el rol de las universidades en los nuevos procesos de integración caracterizados como regionalismo abierto. Es en ese contexto en que se inserta la principal política universitaria promovida y liderada por la institución: la Asociación de Universidades Grupo de Montevideo(AUGM), que configuró un exitoso regionalismo educativo, basado en las macro universidades públicas de los países del MERCOSUR. AUGM constituye desde ese momento y hasta el presente una de las iniciativas más relevantes en la escala regional. ${ }^{22}$

\footnotetext{
${ }^{20}$ Disponible en: http://www.universidad.edu.uy/prensa/renderltem/itemld/31582

${ }^{21}$ Ver: http://udelar.edu.uy/confucio/. Acceso el: 28 abr. 2019.

${ }^{22}$ En ese proceso de transformación interna jugaron un rol clave la exitosa experiencia del PEDECIBA, la creación de las Facultades de Ciencias y de Ciencias Sociales, la creación de Comisiones sectoriales, como la de Investigación Científica, en el marco de políticas públicas que fomentaron el sector de Ciencia, Tecnología e Innovación, más allá de la Udelar.
} 
Por la misma época se conformó el Sector Educativo del Mercosur(SEM), que desarrolló sucesivos Planes Trienales y Planes Estratégicos. La celebración de diversos protocolos y el memorándum de entendimiento de los ministros de Educación-apuntando a la instalación de un mecanismo experimental de acreditación de carreras universitarias de grado (MEXA)- construyeron un marco cierto de interdependencia regional también en el terreno educativo que avanzó en la implementación de un mecanismo de acreditación definitivo: ARCUSUR.

Los aportes del MERCOSUR Educativo incluyen también al Programa MARCA "Movilidad Académica Regional para las carreras acreditadas por el Mecanismo de Acreditación de Carreras de grado en el MERCOSUR": Programa MARCA para la movilidad de Docentes de Grado; Programa de Asociación Universitaria para la Movilidad de Docentes de Grado del MERCOSUR; Programa de Intercambio Académico de Portugués y Español; y al Proyecto "Apoyo de la Unión Europea al Programa de Movilidad MERCOSUR en Educación Superior" cofinanciado por la Unión Europea. También se encuentran en desarrollo los siguientes programas: Sistema Integral de Fomento para la Calidad de los Posgrados del MERCOSUR; Programa de Proyectos Conjuntos de Investigación; Programa de Asociación para el Fortalecimiento de Posgrados; Programa de Formación de Recursos Humanos y la creación del Núcleo de Estudios e Investigaciones en Educación Superior del MERCOSUR. La Decisión CMC №36/12 promovió la constitución del Sistema Integrado de Movilidad del MERCOSUR (SIMM). Sin embargo, existen aún desafíos pendientes en materia de integración regional educativa (ALFA PUENTES, 2012).

Una iniciativa clave de internacionalización es la Red Especializada en Ciencia y Tecnología (RECYT), promovida por la Organización de Estados Iberoamericanos y la Secretaría General Iberoamericana, que por primera vez ha permitido una perspectiva regional sobre política del conocimiento con base en indicadores comparables. Y, en el ámbito institucional del MERCOSUR, resulta altamente relevante la creación del Núcleo de Estudios e Investigación en Educación Superior (NEIES), el que promueve la investigación por medio de concursos regulares de convocatorias a consorcios interuniversitarios y la publicación de una revista especializada que es la "Revista Integración y Conocimiento" (MARTíNEZ LARRECHEA; CHIANCONE, 2013).

En lo interno a la Udelar, la instalación del nuevo Servicio de Relaciones Internacionales (SRI) fue en lo inmediato una respuesta a las necesidades 
de organizar la participación en la CRES 2018 -como una forma estratégica de fortalecer institucionalmente la gestión de la cooperación- sin embargo, los resultados de la CRES 2018 no lograron constatar avances en materia de construcción de un espacio regional que ya había sido anunciado en Cartagena de Indias (CRES, 2008). Podría afirmarse que el proceso de descentralización institucional de la Universidad de la República-con la creación de los Polos de Desarrollo Universitarios (PDU) y la conformación de los Centros Universitarios Regionales- ha completado el escenario de regionalización e internacionalización dada la localización de las nuevas sedes universitarias en franjas de frontera de Uruguay con sus países limítrofes, proceso que aún no se ha analizado lo suficiente desde el punto de vista de la internacionalización, pero que está generando nuevos formatos de convergencia poco estudiados aún (VIERA DUARTE, 2018).

En cuanto a la formalización expresa de una política de internacionalización, si bien Udelar no elaboró todavía un documento estratégico al respecto, el análisis de diversas fuentes documentales de la propia institución permite concluir que la concepción subyacente en ella es la de una internacionalización solidaria o cooperativa, orientada en primer lugar al contexto regional

Como síntesis, podemos entonces insistir en que la Universidad de la República ofreció una primera respuesta estratégica al nuevo contexto regional y global al comenzar los años noventa, con el establecimiento de la AUGM en agosto de 1991; que buena parte de los programas de movilidad se han enfocado a los espacios regionales, latinoamericano e iberoamericano y que en segundo lugar se ha dado prioridad a algunos países europeos-entre los cuales se destacan Alemania, España, Francia, Italia- según el número de convenios celebrados y la cooperación académica en curso.

Los documentos institucionales identifican desafíos pendientes en diversos planos, que forman parte de la agenda universitaria de la internacionalización en el futuro próximo. Las macro universidades públicas de América Latina y del MERCOSUR, constituyen actores de especial relevancia. De la calidad de la respuesta que ellas den a los desafíos de la convergencia, la internacionalización, y la movilidad depende que la región latinoamericana logre conformar un espacio común de conocimiento y de Educación Superior. 


\section{REFERENCIAS}

ALFA PUENTES. La Subregión del MERCOSUR y la Movilidad Académica. Estado de situación, propuestas y orientaciones desde el Proyecto ALFA PUENTES. Alfa Puentes. AUGM, 2012. Disponible en: http://cooperacion.udelar.edu.uy/es/wp-content/uploads/2013/03/subregional-mercosur-.pdf.pdf. Acceso el: 28 abr. 2019.

ALTBACH, P.; KNIGHT, J. Visión panorámica de la internacionalización en la educación superior: motivaciones y realidades. Perfiles Educativos, México, v. 28, n. 112, p. 13-39, ene. 2006. Disponible en: http://www.scielo.org.mx/scielo.php?script=sci_ arttext\&pid=S0185-26982006000200002\&lng=es\&nrm=iso. Acceso el: 8 oct. 2019.

ARTIGAS, S. La Reforma Universitaria (2007-2010): ejes temáticos, actores e influencias. 2014. Tesis (Maestría en Área Social y CSE) - Universidad de la República (Udelar), Montevideo, Uruguay, 2014.

ASOCIACIÓN UNIVERSITARIA GRUPO MONTEVIDEO (AUGM). Página web oficial. Disponible en: http://grupomontevideo.org/sitio/. Acceso el: 3 mar. 2019.

ASCONAVIETA DA SILVA, P. H.; SOUZA LIMA, A. El contexto de los Cursos Binacionales en la Frontera Sant'Ana do Livramento-BR/Rivera-UY. In: DINIS PEREIRA, M. A. P.; MOURA DE MELLO, L. (Org.). Cursos binacionais: relatos de uma experiência. Santana do Livramento, RS, Brasil: Cia do eBook, 2015. p. 9-21.

BIZZÓZERO, L.; ABREU, S. Los países pequeños: su rol en los procesos de integración. Montevideo, Uruguay: Bid-intal, 2000. v. 8.

BIZZÓZERO, L. Internacionalización de la educación superior y mejora de las funciones en la Universidad. Escuela de Invierno. Núcleo de Evaluación Institucional, Planeamiento Estratégico y Gestión Universitaria de AUGM, 10-14 de setiembre, 2018. Disponible en: http://gestion.udelar.edu.uy/planeamiento/wp-content/uploads/sites/33/2018/11/4Lincoln-Bizzozero-Internacionalizaci\%C3\%B3n-de-la-educaci\%C3\%B3n-superior-y-mejorade-las-funciones.pdf. Acceso el: 3 mar. 2019.

BIZZOZERO, L.; HERMO, J.; MARTínEZ LARRECHEA, E. Proyecto: estrategias de internacionalización de las universidades del Mercosur en la globalización. Núcleo de Estudios e Investigaciones en Educación Superior del Sector Educativo del MERCOSUR. In: JORNADAS DE INVESTIGACIÓN, 13., 15-17 setiembre 2014. Montevideo, Uruguay: Facultad de Ciencias Sociales: UdelaR, 2014. 
CRES. Declaración de la III CONFERENCIA REGIONAL DE EDUCACIÓN SUPERIOR PARA AMÉRICA LATINA Y EL CARIBE. Córdoba, Argentina, 14 de junio de 2018. Disponible en: http://www.iesalc.unesco.org/2018/12/13/informe-general-de-la-cres-2018/. Acceso el: 30 oct. 2019.

DGRC. Página web del Servicio de Relaciones Internacionales de Udelar. 2019. Disponible en: http://cooperacion.udelar.edu.uy/es/?page_id=172. Acceso el: 3 mar. 2019.

DGRC. Informe internacionalización. Políticas institucionales en cooperación regional e internacional. CRI. Sala Maggiolo. Montevideo: Udelar, mayo 2009. Disponible en: http://cooperacion.udelar.edu.uy/es/wp-content/uploads/2013/03/Informe-deinternacionalizaci\%C3\%B3n-actualizado-para-p\%C3\%A1gina-web-20140519.pdf. Acceso el: 10 abr. 2019.

DIARIO NORTE. Nota de prensa, 11 de mayo de 2018. Disponible en: https://www. diarionorte.com.uy/sociedad/reunion-binacional-apunta-al-desarrollo-de-la-educacionterciaria-en-la-frontera-44879.html. Acceso el: 3 mar. 2019.

DINIS, M. A. P.; MOURA DE MELLO, L. (Org.). Cursos binacionais: relatos de uma experiência. Santana do Livramento, RS, Brasil: Cia do eBook, 2015. Disponible en: https:// www.researchgate.net/publication/283644603_Cursos_binacionais_relatos_de_uma_ experiencia_inovadora. Acceso el: 10 abr. 2018.

GONZÁLEZ, L. Informe sobre el desarrollo de la extensión y actividades en el medio en frontera. Rivera: CUR-Udelar, 2018.

IDR. Encuentros de frontera. Rivera: IDR, 2018. Disponible en: http://www.rivera.gub.uy/ portal/tag/frontera/. Acceso el: 10 abr. 2019.

KNIGHT, J. Internationalization: elements and checkpoints. CBIE Research n. 7. Canadian Bureau for International Education (CBIE)/Bureau canadien de l'éducation internationale (BCEI). 220 Laurier Avenue West, Suite 1550, Ottawa, Ontario K1P 5Z9, 1994.

MARTÍNEZ LARRECHEA, E.; CHIANCONE, A. Hacia una nueva estrategia de integración educativa, Revista Integración y Conocimiento. Revista del Núcleo Interdisciplinario de Educación Superior, Buenos Aires, n. 2, 2013. Disponible en: http://revistas.unc.edu.ar/ index.php/integracionyconocimiento/article/view/5701/6914. Acceso el: 10 abr. 2019.

MIRANDA, E. M. La universidad en la sociedad del conocimiento. In: MOROSINI, M. (Org.). Foro Latinoamericano de Educación Superior (FLAES). São Carlos. SP: Pixel, 2014. 
MOROSINI, M. Integração e internacionalização da Educação Superior. In: MOROSINI, M. (Org.). Foro Latinoamericano de Educación Superior (FLAES). São Carlos. SP: Pixel, 2014.

OLVERA, R.; VIERA, P. Internacionalización de la Educación. Una experiencia educativa en zona de frontera: Oportunidades y desafíos. In: DINIS PEREIRA, M. A. P.; MOURA DE MELLO, L. (Org.). Cursos binacionais: relatos de uma experiência. Santana do Livramento, RS, Brasil: Cia do eBook, 2015. p. 87-95. Disponible en: https://www.researchgate.net/ publication/283644603_Cursos_binacionais_relatos_de_uma_experiencia_inovadora. Acceso el: 10 abr. 2019.

UDELAR. La política de regionalización y descentralización de UdelaR 2007-2011. Montevideo, Uruguay: Udelar, 2012. (Colección Hacia la Reforma Universitaria n. 14).

UDELAR. Dirección General de Relaciones y Cooperación. Página web oficial. Disponible en: http://cooperacion.udelar.edu.uy/es/. Acceso el 28 feb. 2020.

UDELAR-CCI. Logros de la UdelaR en el interior del país (2005-20015). Montevideo, Uruguay: Baferil, 2015.

UDELAR-DGRC. Gráficos de datos estadísticos. Montevideo, Uruguay: Udelar, 2014. Disponible en: http://cooperacion.udelar.edu.uy/es/wp-content/uploads/2012/09/ Gr\%C3\%A1ficos-de-Datos-estad\%C3\%ADsticos-para-p\%C3\%A1gina-web2.pdf. Acceso el: 15 abr. 2018.

UNESCO. Conferencia Mundial sobre la Educación Superior 2009: La nueva dinámica de la educación superior y la investigación para el cambio social y el desarrollo. París, 2009

VIERA-DUARTE, P. Nuevos formatos de convergencias de la educación superior: investigación en ciudades binacionales del Cono Sur de América Latina. Revista Internacional de Educação Superior, Campinas, SP, v. 4, n. 2, p. 330-46, maio/ago. 2018. DOI: https://doi.org/10.20396/riesup.v4i2.8650708

VIERA-DUARTE, P. Estudios sobre políticas de democratización e internacionalización de la Educación Superior en regiones de frontera: reflexiones metodológicas. In: MAZZEI, Enrique. I Encuentro de Investigadores del Área Social de la Región Noreste de la Universidad de la República. Montevideo, Uruguay: CCI-CEF- UdelaR, 2017. 


\section{Referencias digitales}

Udelar. http://cooperacion.udelar.edu.uy/es/

Udelar. http://cooperacion.udelar.edu.uy/es/wp-content/uploads/2012/09/OrganigramaRelaciones-y-Cooperaci\%C3\%B3n-aprobado-2012-1.pdf

Udelar. http://cooperacion.udelar.edu.uy/es/wp-content/uploads/2013/03/Informe-deinternacionalizaci\%C3\%B3n-actualizado-para-p\%C3\%A1gina-web-20140519.pdf

\section{Sobre los autores:}

Patricia Viera-Duarte: Doctora en Educación. Máster en Educación con énfasis en Investigación de procesos de enseñanza y aprendizaje. Diplomada en Diseño y Desarrollo Curricular. Licenciada en Educación. Maestra de Educación Primaria. Miembro activo del Sistema Nacional de Investigadores (SNI) de la Agencia Nacional de Investigación e Innovación (ANII) de Uruguay. E-mail: pviera99@gmail.com, Orcid: https://orcid.org/0000-0002-1134-7216

Adriana Chiancone: Doctora en Estudios Sociales de la Ciencia y la Tecnología - Magister en Ciencias Sociales - Licenciada en Antropología Social Investigadora SNI, Uruguay. E-mail: achiancouniversidad@gmail.com, Orcid: https://orcid.org/0000-0002-4332-3702

Enrique Martínez Larrechea: Doctor en Relaciones Internacionales. Magíster en Ciencias Sociales. Licenciado en Sociología. Investigador SNI, Uruguay. E-mail: martinez.larrechea@gmail.com, Orcid: https://orcid.org/0000-0001-7453-2796

\section{Recibido el 10 de noviembre de 2019.}

Aprobado el 18 de febrero de 2020. 
